\title{
Analysis and application of whispering gallery modes of the triple-layer-coated microsphere resonator
}

\author{
Mengyu Wang, Xueying Jin, Fei Li, and Keyi Wang* \\ Department of Precision Machinery and Precision Instrumentation, University of Science and \\ Technology of China, Hefei 230027, P.R China
}

\begin{abstract}
Microsphere resonators supporting whispering gallery modes (WGMs) have been extensively applied for considerable fields including narrow linewidth filters, high-sensitivity sensors, and nonlinear optics. We numerically demonstrate a coated microsphere resonator with three layers of high, low, high refractive-index (RI) from inside to outside. A phase matched waveguide is used to overlap the WGMs evanescent radiation field. Eigen-mode, relative intensity spectra, electromagnetic (EM) field distributions are observed to analyze resonant characteristics of WGMs by using the finite difference time domain method. As a result, two brilliant rings with strong EM fields distribute in two high-RI layers. By optimizing the gap distance between the microsphere and waveguide, the WGMs of two high-RI layer are efficiently excited. More energy is stored in such a structure rather than a single-layer-coated microsphere. Our approach provides the RI sensing application with such a triple-layer-coated structure.
\end{abstract}

\section{Introduction}

Optical microresonators supporting whispering gallery modes (WGMs) have attracted increasing attention in numerous fields, such as narrow linewidth filters, biosensors, microlasers [1]. These geometries are typically: spheres, disks, toroids, or bubbles. WGMs in micro-resonators guide strongly light waves circulates about the equator of the resonator geometry by continuous total internal reflection, especially the microspheres, leading to quality $(Q)$ factors in excess of $10^{9}$ being achieved [2]. Due to the advantages of high $Q$ factors and low mode volumes, much optical effect in laser process can be remarkably enhanced. Hence, microsphere resonators are widely studied. Coating of microsphere resonators is a very promising technique for optimizing their properties and preserving the high- $Q$ factor. For instance, I. Teraoka et al confirmed that coating a microsphere resonator with a high RI layer could expose a stronger evanescent field [3]. They further calculated a multilayer-coated microsphere and discovered each of two high-RI layers could sustain its own WGM [4]. In this paper, we will investigate microsphere resonator's WGM properties. Then, we simulate WGMs of the triple-layer-coated microsphere resonator with three layers

\footnotetext{
* Corresponding author: kywang@ustc.edu.cn
} 
of high, low, and high RIs. Compared to a single-layer microsphere, a three-layer microsphere can store more energy. At last, an application in RI sensing field is demonstrated.

\section{Theoretical and numerical model}

\subsection{Whispering gallery modes}

WGMs are characterized by three quantum indices $(l, m, n)$, where $l, m, n$ represent the EM field component along radial, angular, and azimuthal direction, respectively. In the spherical polar coordinates $(r, \theta, \varphi)$, for a steady-state WGM at resonance, microsphere resonances are found as roots of a transcendental equation of transverse electric (TE) polarization and the electric field $S(r)$ for different $l$ on radical distribution [3] can be expressed as equation (1):

$$
S(r)= \begin{cases}A_{l} \cdot \Psi_{l}\left(n_{1} k r\right) & r<R \\ B_{l} \cdot \chi_{l}\left(n_{0} k r\right) & r \geq R\end{cases}
$$

where $n_{1}$ and $n_{0}$ stand for the RIs of the microsphere and surrounding medium. $R$ is the radius of microsphere. $k$ is the wave number in free space. The coefficients $\left(A_{l}, B_{l}\right)$ is determined by the continuity of boundary conditions: $A_{l} \cdot \psi_{l}\left(n_{1} k R\right)=B_{l} \cdot \chi_{l}\left(n_{0} k R\right)$. Here, $\psi_{l}(z) \equiv z j_{l}(z)$ and $\chi_{l}(z) \equiv z n_{l}(z)$ are spherical Ricatti-Bessel and Ricatti-Neumann functions, where $j_{l}(z)$ and $n_{l}(z)$ stand for the spherical Bessel and Neumann functions of first kind. The resonance condition can be given as:

$$
\frac{n_{1}}{n_{0}} \frac{\dot{\Psi}_{l}\left(n_{1} k R\right)}{\psi_{l}\left(n_{1} k R\right)}=\frac{\dot{x}_{l}\left(n_{0} k R\right)}{x_{l}\left(n_{0} k R\right)}
$$

The electrical field $X(r)$ on the equator plane can be given as: $X(r)=$ $N_{s} H_{m}(\sqrt{m} \theta) \cdot \exp \left(-m \theta^{2} / 2\right) \cdot S(r) . H_{m}(z)$ is the Hermitian function, $N_{s}$ is normalisation constant, and $\theta$ is the angular angle. The electrical field $W(r)$ in the microsphere surface can be written as: $W(r)=X(r) \cdot \exp ( \pm j n \varphi) \cdot \varphi$ is the azimuthal angle. For given $(l, m, n)$, the WGMs (TE) of a silica microsphere resonator can be calculated using MATLAB. Normalized modal distributions of microsphere resonators with $(l, m, n)=(1,25,25)$, and $(2,25,24)$ can be shown as figure $1 . l$ and $m-n$ determine the distribution homogeneity of WGMs and stand for the peaks on the radial distribution and on the azimuthal direction. The most confined mode $(l=1, m-n=0)$ is more conscious and is called the fundamental mode of WGMs.
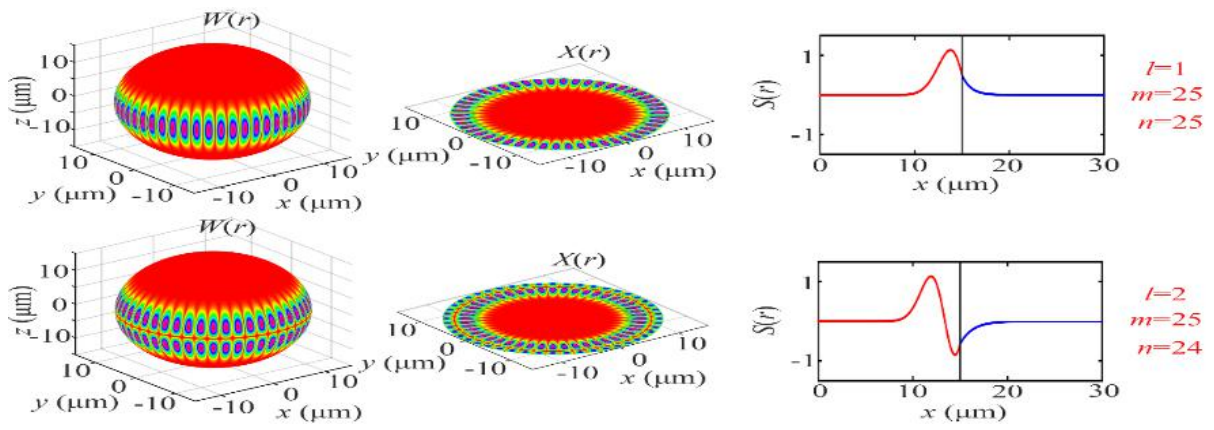

Fig. 1. Electrical field on the surface of the microsphere, on the equator plane, and on the radial distribution of TE mode of a silica microsphere $(R=15 \mu \mathrm{m})$ with $(l, m, n)=(1,25,25)$, and $(2,25,24)$. 


\subsection{Numerical model and theory}

The most confined mode realize high photon degeneracy and small mode volume. However, the fundamental mode is difficult to be excited by near-field coupling. Therefore, we use the coated method to ameliorate the challenge of energy dispersion in other WGMs. Here, we calculate them by numerical computation using FDTD method [5]. We present a WGM in a silica microsphere coated with three layers. Figure 2(a) shows our simulation model of triple-layer-coated microsphere resonator. The thickness widths of three layers $(A, B, C)$ are $d_{A}, d_{B}$ and $d_{C}$, and the RIs of three layers are $n_{A}, n_{B}, n_{C}$. The total radius of the microsphere is $R$. The principle of the triple-coated microspheres are analyzed by using Teraoka's numerical method $[3,4]$. The electric field $S(r)$ of WGMs on the radial distribution is written as:

$$
=\left\{\begin{array}{cc}
A_{l} \Psi_{l}\left(n_{1} k r\right) & r<R-d_{A}-d_{B}-d_{C} \\
C_{l 1} \psi_{l}\left(n_{C} k r\right)+D_{l 1} \chi_{l}\left(n_{C} k r\right) & R-d_{A}-d_{B}-d_{C}<r<R-d_{A}-d_{B} \\
C_{l 2} \Psi_{l}\left(n_{B} k r\right)+D_{l 2} \chi_{l}\left(n_{B} k r\right) & R-d_{A}-d_{B}<r<R-d_{A} \\
C_{l 3} \psi_{l}\left(n_{A} k r\right)+D_{l 3} \chi_{l}\left(n_{A} k r\right) & R-d_{A}<r<R \\
B_{l} \chi_{l}\left(n_{0} k r\right) & r>R
\end{array}\right.
$$

where the coefficients $A_{l}, B_{l l}, C_{l l}, C_{l 2}, C_{l 3}, D_{l l}, D_{l 2}, D_{l 2}, D_{l 3}$ are determined by the boundary conditions at $r=R-d_{A}$ and $r=R-d_{A}-d_{B}$, where $S(r)$ and $S(r)$ be continuous across these interfaces. Applying them for TE polarization, the resonance condition was given as:

$$
\frac{n_{B}}{n_{A}} \frac{\left(C_{l 2} / D_{l 2}\right) \dot{\Psi}_{l}\left(z_{B 2}\right)+\dot{x}_{l}\left(z_{B 2}\right)}{\left({ }{ }_{l 2} / D_{l 2}\right) \Psi_{l}\left(z_{B 2}\right)+x_{l}\left(z_{B 2}\right)}=\frac{\left(C_{l 3} / D_{l 3}\right)}{\left({ }_{l 3} / \dot{\Psi}_{l}\left(z_{A 1}\right)+\dot{x}_{l}\left(z_{A 1}\right)\right.}
$$

where $z_{A 1}, z_{B 2}$ are given by: $z_{A 1}=n_{A} k\left(R-d_{A}\right), z_{B 2}=n_{B} k\left(R-d_{A}-d_{B}\right)$. Likewise, we work out the electrical field $X(r), W(r)$ on the equator plane and in the microsphere surface.

The light in WGMs is tightly confined. The coupling efficiency can reach the highest value (almost 100\%) with the tapered fiber waveguide [6]. Hence, in our numerical mode shown in figure 2(b), we add a fiber waveguide to overlap the WGMs evanescent field of the coated microsphere. The microsphere consists of silica glass and has a RI of $n_{l}=1.46$. The overall radius of the microsphere with three-layer coatings is $R=3.5 \mu \mathrm{m}$. The fiber waveguide with a thickness of $d_{\mathrm{w}}=300 \mathrm{~nm}$. The gap distance between the waveguide and microsphere is $g$. The surrounding medium is set to $n_{0}=1$. The pump laser for TE mode varies between $1 \mu \mathrm{m} \sim 1.2 \mu \mathrm{m}$ is employed through the waveguide in the leftmost position. The coupling light can be exported into Photo Detector (PD) in the position $(D)$ and the rightmost position.
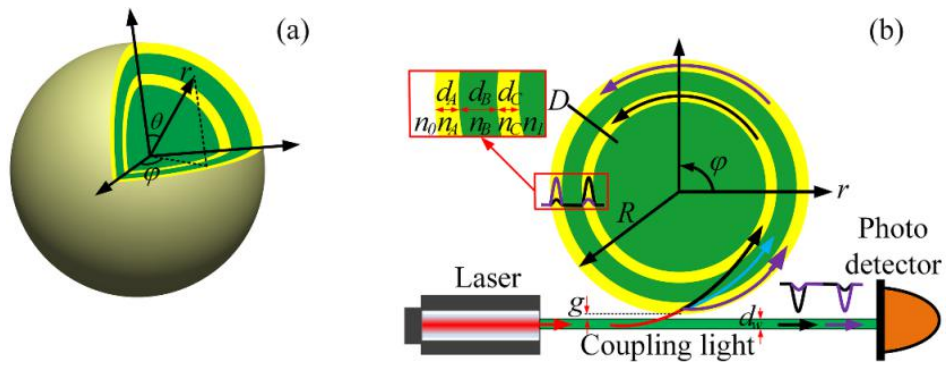

Fig. 2. Triple-layer-coated silica microsphere and 2D FDTD simulation model.

\section{Results and discussion}


To study the resonance properties of WGMs in the triple-layer-coated microsphere, we explore eigen-mode distribution at first. Distic et al had a beautiful demonstration of a microsphere coated silicon [7]. The RI difference of the microsphere $(n=1.46)$ and the coated layer $(n=3.58)$ can effectively enhance the energy density and alleviate the highorder WGMs. Here, the inner layer $(C)$ coats silicon material. To increase the RI difference further, we add the middle layer $(B)$ coated silica material and the outer layer $(A)$ coated silicon material. The three layer structure (silicon-silica-silicon) will confine more light. We set the thickness of layer $B$ to $d_{B}=400 \mathrm{~nm}$ and the thickness of layer $A$ and $C$ to $d_{A}=d_{C}=100 \sim 300 \mathrm{~nm}$ for the purpose of the width value range containing the first radial WGMs $(l=1)$. The WGMs' eigen-mode field of the triple-layer-coated structure can be shown in figure $3(\mathrm{a})$. When $d_{A}+d_{B}+d_{C}=800 \mathrm{~nm}$ and $d_{B}=400 \mathrm{~nm}$ remain unchanged, three cases $\left(d_{A}=100 \mathrm{~nm}, d_{A}=200 \mathrm{~nm}, d_{A}=300 \mathrm{~nm}\right)$ can be calculated. Two different radial modes can be found on two high-RI layers, which be called as 'coastal mode' and 'inland mode' in Ref [4], we call them 'outer mode' (OM) and 'inner mode' (IM) shown in figure 3(b). For $d_{A}=100 \mathrm{~nm}\left(d_{C}=300 \mathrm{~nm}\right)$, we discover the electric field energy for the IM appears dispersive, which means the peak range of the first radial WGMs is wide. The result will lead to the reducing of energy density. Meanwhile, the electric field energy for the OM don't remain the centre of the outer layer. More overflowing energy will result in a low $Q$ factor. Similarly, for $d_{C}=100 \mathrm{~nm}\left(d_{A}=300 \mathrm{~nm}\right)$, the energy density and $Q$ factor are lower than the case for $d_{C}=200 \mathrm{~nm}\left(d_{A}=200 \mathrm{~nm}\right)$. In such a structure, we conclude that the IM and $\mathrm{OM}$ had a near linear dependence on the thickness of the two high-RI layers, especially when $d_{\mathrm{A}}=d_{C}=200 \mathrm{~nm}$.
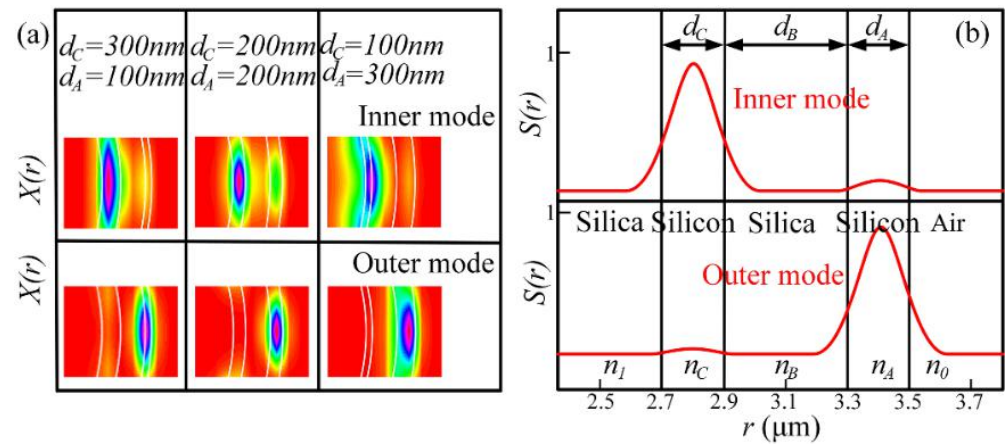

Fig. 3. (a) Electric field distributions of different coating layers; (b) Inner mode and outer mode along the radial direction with the width of three layers $d_{A}=200 \mathrm{~nm}, d_{B}=400 \mathrm{~nm}, d_{C}=200 \mathrm{~nm}$.

Once the eigen-mode of WGMs are calculated, we discovered these features that the resonance intensity of three-layer only could be excited in the inner and outer layers, and resonant wavelengths separate just as the coupling in two WGMs resonators. The result also confirms the conclusion in the previous section. The OM and IM mode in two high-RI layers can be excited by waveguide coupling. The three-layer structure will increase the highest maximum stored energy and relative intensity to a larger degree in the same mode volume. The coupling strength can depend on two parameters. The first parameter is the overlap of the evanescent fields. The second parameter is the phase-matching between the WGMs in the microsphere and the propagating mode of the waveguide. The phase matching condition is primarily can not be changed once the sphere is produced. We set the width of the fiber waveguide is $300 \mathrm{~nm}$. It is thick enough support the TE mode, leading to the complete confinement effect inside the coatings. To excite the WGMs efficiently, the phase matching condition can be optimized by changing the gap width $(g)$. As the gap increases, we can obtain the resonance relative intensity spectra in four cases, as showed in figure 4. By changing the gap distance, we noticed that the resonance wavelength shift and 
the number modify of the resonance. As a result, the resonance pairing disappears and resonance number grows as the gap increases. The coupling between the WGM in the highRI layer and the mode in the waveguide can be optimized by changing the gap. For $g=200$ $\mathrm{nm}$, the WGMs of two high-RI layers can be efficiently excited. In addition, we notice that the full width at half-maximum (FWHM) of the resonator grow wider with the increasing wavelength. The result may be a consequence of the larger overlap of the waveguide field and WGMs field. The mode overlap will bring about a larger coupling loss, which increased the FWHM of WGMs.
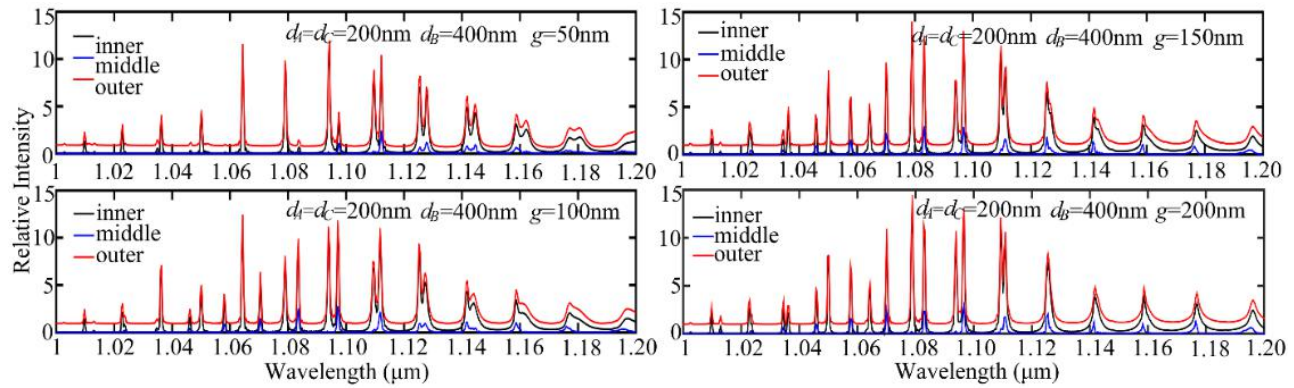

Fig. 4. Resonance intensity spectra of triple-layer-coated microsphere for different coupling gaps between the microsphere and waveguide with $g=50 \mathrm{~nm}, 100 \mathrm{~nm}, 150 \mathrm{~nm}, 200 \mathrm{~nm}$, respectively.

We further explored the EM field of three-layer coatings at these resonance wavelengths in figure 4. The on-resonance and off-resonance characteristics were illuminated to demonstrate the great difference of the electric field for imported excitation wavelengths in figure 5. Diverse cases under outer layer resonance and inner layer resonance were compared. The EM field distributions can also represent the energy distributions in the microsphere surface. The two high RI layers, are guided for those wavelengths examined, so they form a kind of directional coupler where the light localized in each of them beats with the other. This led to the very beautiful results of figure 5 , where light was trapped in a two-fold waveguide. A very brilliant ring with strong electric field could also be found only in the inner and outer layers. This is consistent with the observation from the resonance intensity of the inner and outer layers, as given in figure 4 . The phenomenon confirmed that the result in the previous section further. From two cases in figure 5 (a) and (d), less energy of pump laser can couple into the outer and inner layer and most energy remain in the waveguide. This is a consequence of the phase mismatching of the coupling. For four cases in figure 5 (b), (c) and (e), (f), most energy of pump laser can couple into the layer and little energy remain in the waveguide. This is a consequence of perfect phase matching of the coupling. The energy density difference in four cases can be explained as different the EM field component along the angular direction $m$ on the equator plane. From three cases in figure $5(\mathrm{~g}),(\mathrm{h})$ and (i), the outer and inner layer resonance can be excited at the same time because of the WGM mode overlap. The coupling efficiency is low and the energy is dispersive.

On one hand, to match phase, we calculate the best parameters: $d_{w}=300 \mathrm{~nm}, g=200 \mathrm{~nm}$. As a result of phase matching, WGMs of high-RI layers can be efficiently excited. On the other hand, the distance between two high-RI layers, also $d_{B}$, can be optimized to obtain two strong resonance mode in two high-RI layers. To further optimize the triple-layer resonance structure and study the impact of the coating thickness, we solve the stored energy $\left(E_{n}=\int \varepsilon(r) E^{2}(r) d r^{3}\right)$ by changing low-RI layer thickness $d_{B}$, as shown in figure $6(\mathrm{a})$, where $E^{2}(r)$ is the electric fields intensity, $\varepsilon(r)$ is the electric permittivity. The highest stored energy can be obtained when $d_{B}=275 \mathrm{~nm} \sim 325 \mathrm{~nm}$. For $d_{B}<275 \mathrm{~nm}$, the small RI $n_{B}$ of the intervening layer could not hold a WGM. For $d_{B}>325 \mathrm{~nm}$, the mode bonding in two 
high-RI layers is broken, $E_{n}$ will decrease with an increasing $d_{B}$. After optimizing the triplelayer structure $\left(d_{A}=200 \mathrm{~nm}, d_{C}=200 \mathrm{~nm}, d_{B}=300 \mathrm{~nm}\right)$, its stored energy is almost 1.6 times higher than that of single high-RI layer structure (the coated layer $d=200 \mathrm{~nm}$ ), as shown in figure $6(\mathrm{~b})$. The unique structure would expand the performance of light-matter interaction in lasers applications. Figure 6(c) shows the typical resonant dip in a transmission spectrum for $d_{B}=300 \mathrm{~nm}$. $Q$ factor is one of the most important properties in any kind of geometrical resonator. $Q$-factors are a direct measure of the ability of the cavity to confine and store light. In our work, we cannot determine $Q$ from the FWHM [8] ( $Q=\lambda / \Delta \lambda=1253 / 0.35=3580$ ) as shown in figure 6(c) because the FWHM of each resonance in the spectrum is limited by the time of simulation. Instead, $Q$ factor should be determined by the slope of the envelope of the decaying signal using the formula: $Q=-$ $2 \pi f_{0} \log _{10}(e) / k=10591.5$, where $f_{0}$ is the resonant frequency of the WGM, and $k$ is the slope of the log of the time signal envelope.

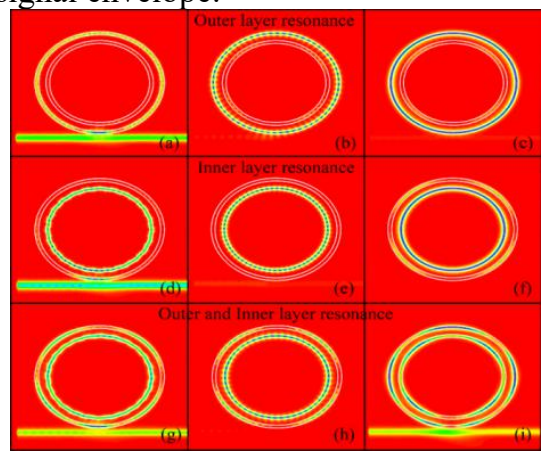

Fig. 5. Typical magnetic field profiles for outer layer, inner layer, and outer and inner layer resonance.
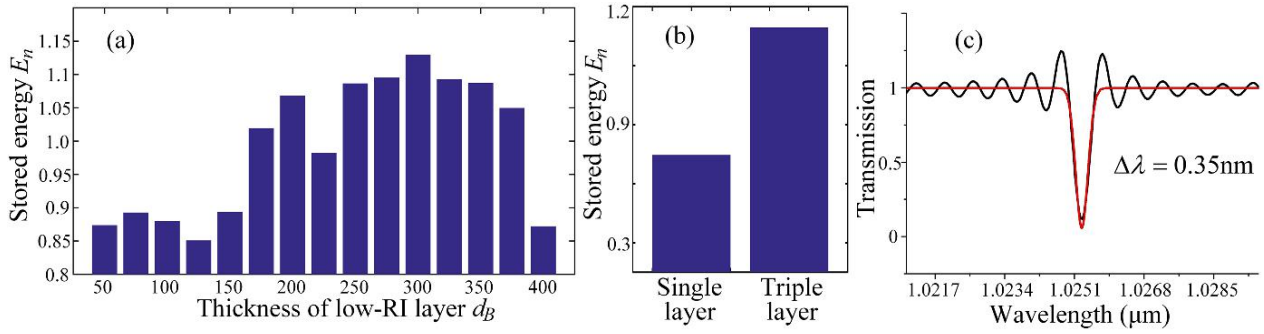

Fig. 6. (a) Stored energy as a function of the thickness of two high-RI layers. (b) Comparison between single-layer and triple-layer structure. (c) Typical resonant dip.

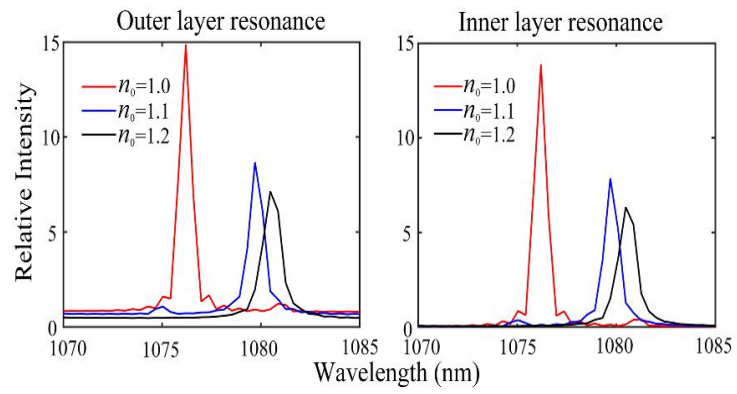

Fig. 7. Relative intensity spectra for different surrounding medium RIs: $n_{0}=1.0,1.1,1.2$, respectively.

At last, we investigate the influence of the surrounding medium on coupling characteristics. We obtain the relative intensity spectra with varying excitation for different cases of $n_{0}=1.0, n_{0}=1.1$ and $n_{0}=1.2$ as shown in figure 7 . The response to an increasement 
from 1.0 to 1.1 in the surrounding medium RI is an obvious resonance wavelength upshift of $3.6 \mathrm{~nm}$ for the outer and inner WGM resonance. However, the response to an increasement from 1.1 to 1.2 in the surrounding medium RI is a slight resonance wavelength upshift of $0.8 \mathrm{~nm}$. According to the equation of critical angle, $\theta_{C}=\sin ^{-1}\left(n_{C} / n_{0}\right)$, $\theta_{C}$ can get larger as $n_{0}$ increases, and the resonant frequency will decrease as a result of the enlargement of the effective traveling distance of the photons in a resonance mode, meaning that calls for a bigger wavelength light to match the resonance condition to maintain the same resonance mode. The response can be also explained as an increasement of the effective RI $n_{\text {eff. }}$. According to the resonance equation $\left(2 \pi R n_{\text {eff }}=m \lambda_{0}\right)$, the wavelength

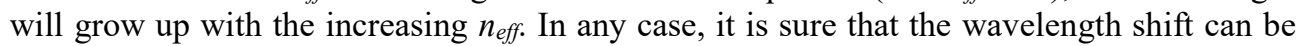
used as RI sensors. The measure of double layer will increase the sensibility of RI sensor. Accordingly, our results suggest a potential RI sensor field and the possibility of changing the surrounding medium is particularly worthy of academic investigation by comparing numerous key medium indicators.

\section{Conclusion}

In summary, the characteristics of WGMs in a triple-coated microsphere have been analyzed and optimized. The WGMs of two high-RI layers were perfectly excited by a phase matched waveguide. For an appropriate coating thickness, energy was stored at the maximum. $Q$ factor of WGMs was calculated by the slope of the envelope. It would be a good method to increase light-matter interaction. The triple-layer-coated microsphere can be used in the RI sensing.

\section{References}

1. K. J. Vahala, “Optical microcavities," Nature 424, 839 (2003)

2. A. A. Savchenkov, M. L. Gorodetsky, V. S. Ilchenko, "Ultimate Q of optical microsphere resonators," Opt lett 21, 453-55 (1996)

3. I. Teraoka, S. Arnold, "Enhancing the sensitivity of a whispering-gallery mode microsphere sensor by a high-refractive-index surface layer," J OSA B 23, 1434-41 (2006)

4. I. Teraoka, S. Arnold, "Coupled whispering gallery modes in a multilayer-coated microsphere," Opt lett 32, 1147 (2007)

5. J. M. Hall, V.S. Afshar, M.R. Henderson, et al, "Method for predicting whispering gallery mode spectra of spherical microresonators," Opt Express 23, 9924-37 (2015)

6. J. C. Knight, G. Cheung, F. Jacques, et al, "Phase-matched excitation of whisperinggallery-mode resonances by a fiber taper," Opt lett 22, 1129-31 (1997)

7. D. Ristic, A. Chiappini, M. Mazzola, et al, "Whispering gallery mode profiles in a coated microsphere [J]. Eur Phys J A 223, 1959-69 (2014)

8. L. K. Kosma, G. Zito, K. Schusteret, "Whispering gallery mode microsphere resonator integrated inside a microstructured optical fiber," Opt Lett 38, 1301 (2013) 